\title{
APPROCHING THE TWIN PRIME CONSTANT
}

\author{
Ibrahima Gueye \\ Ouakam, Quarter Gouye Sor, Dakar - Sénégal \\ ibrahimaeygue@yahoo.fr
}

Keywords: Prime numbers, twin primes, additive theory

\begin{abstract}
In this article I highlight some curiosities about the primes. THROUGH a new conjecture, I think that the twin prime constant can be approached with an easy method.
\end{abstract}

\section{Introduction:}

A prime number is an integer that has exactly two distinct divisors and positive integers are 1 and itself. This definition excludes 1 , which has only one positive integer divisor, it also excludes 0 , which is divisible by all positive integers. It is known since antiquity that there are infinitely many primes. Two twin primes are two primes that differ by only 2. Except for the pair $(2,3)$, this distance of 2 is the smallest possible distance between two prime numbers. The smallest twin primes are 3 and 5, 5 and 7, 11 and 13. The study of distribution and the search for formulas of prime numbers has always interested mathematicians.

* Primes seem to appear in great disorder $2,3,5,7,11,13,17.19, \ldots$ However, the properties stated in the nineteenth century show a behavior not "anarchic". The theorem of prime numbers dwindling by Jacques Hadamard and de la Vallee Poussin Jean teaches us by example that the number of primes less than or equal to $n$ is about $n / \ln n$ ( $\ln n$ where denotes the natural logarithm of $n$ ). This is an indication that the numbers premiersn'arrivent not in absolute chaos but rather, with some regularity.

Prime numbers follow laws that mathematicians are trying to identify. Evidence that such laws exist is that we can put the primes in formulas. Of course, no simple formula is not appropriate and we show by example that no polynomial function gives only prime numbers except in the trivial case where the formula is constant. In 1947, W. Mills surprised the mathematical community by establishing the existence of a constant A which, inserted into a formula, gives a prime number for all $\mathrm{n}$ greater than or equal to 1 . The usefulness of this formula is, however illusory, because we can calculate the constant A only if the primes already know. Another approach illuminates the phenomenon where the $n$th prime number for all $n$ greater than or equal to 1 . Formulas for finding prime numbers must be limited to those that do not involve real constants.

Mathematicians have devised Minac Willans and a more remarkable still, the explanation is less straightforward, but this time, gives all the prime numbers in order and without repetition. This formula contains only 52 symbols! It was not known that such a formula could exist before its publication in 1995. But is it really useful? No, because the implementation of such formulas proves very time consuming to load and no computer program currently uses these formulas that ultimately appear as mathematical games of skill. The real problem is to define computational algorithms (mathematical-computer language) that will quickly prime numbers. Such programs are applicable to cryptography for example.

Much progress has been made in recent years in the handling and knowledge of prime numbers: Probabilistic algorithms for testing the primality identify prime numbers of 100000 digits in fractions of seconds. The factoring algorithms are becoming every year more and more powerful. However, there are no known method to quickly generate a prime number of length $\mathrm{n}$. We see, work on prime numbers and their relationships with computers and many are far from being all explored. Many questions remain to be resolved. Basic research applications joined to build a science of computer networks and communications of tomorrow whose security is today closely linked to prime numbers (cryptography, error correcting codes, distributed algorithms, etc..). 


\section{FORMS TO INFINITY ...}

- No polynomial function $\mathrm{f}(\mathrm{n}): \mathrm{f}(\mathrm{n})=\operatorname{apnexp}(\mathrm{p})+\operatorname{ap}-1 \exp (\mathrm{p}-1)+\ldots+\operatorname{ainexp}(\mathrm{i})+\ldots$ A1n ++ $\mathrm{a} 0$, where $\mathrm{I}$ is an integer equal to 0 and $\mathrm{AD}$ only gives prime numbers for $\mathrm{n}$ positive or zero except in the trivial case where the formula is constant $(\mathrm{f}(\mathrm{n})=\mathrm{a} 0$ with $\mathrm{a} 0$ a prime number).

- Form W. Mills: [A3N] where the bracket [] denotes the integer function or rounded towards zero. This constant - so-called Mills - was calculated with good accuracy and is: $1.306377883863080690468614492602605712916784585156713644368053759966434 \ldots$

- Third formula: [L x 10nexp (2)] - [L x 10exp (n-1) $\left.{ }^{2}\right]$ 10exp (2n-1) indeed gives the nth prime number for all $\mathrm{n}$ other than $1: 2=\mathrm{p} 1$,

$\mathrm{p} 2=3, \mathrm{p} 3=5, \mathrm{p} 4=7,11=\mathrm{p} 5, \mathrm{p} 6=13, \mathrm{p} 7=17$, etc.. The constant contains the numbers hidden in its first decimal, but this time explicitly: $\mathrm{L}=0.20030000500000070000000110000000001300 \ldots$ (The $\mathrm{nth}$ prime number is placed in position $\mathrm{n} 2$ ).

- Form and Minac Willans: It is of little interest in practice [1] [2].

- The search for formulas for prime numbers must be limited to formulas that do not involve real constants that allow too easy to cheat. There are and here is fairly simple:

$$
\begin{gathered}
\mathrm{n}+1 \\
\mathrm{t}(\mathrm{n})+\mathrm{n}=2\left[1 /\left(1+\sum[(\mathrm{n}+2) / \mathrm{p}-[(\mathrm{n}+1) / \mathrm{p}]]\right)\right] \mathrm{n} \geq 0 \\
\mathrm{p}=2
\end{gathered}
$$

This formula gives only prime numbers and gives all, but slowly and repeating Feb. 1 many times: $t$ $(0)=2, \mathrm{t}(1)=3, \mathrm{t}(2)=2, \mathrm{t}(3)=5$,

$\mathrm{t}(4)=2, \mathrm{t}(5)=7, \mathrm{t}(6)=2, \mathrm{t}(7)=2, \mathrm{t}(8)=2, \mathrm{t}(9)=11, \mathrm{t}(10)=2$,

$\mathrm{t}(11)=13, \mathrm{t}(12)=2, \mathrm{t}(13)=2, \mathrm{t}(14)=2$, etc..

Theorem 1: Theorem Wilson

Statement: An integer $\mathrm{p}$ strictly greater than 1 , is a prime number if and only if divides $(\mathrm{p}-1) !+1$.

Theorem 2: Theorem Clement

Statement: For any integer, the pair $(\mathrm{m}, \mathrm{m}+2)$ consists of twin primes if and only if $4[(m-1) !+1]+m \equiv 0 \quad \bmod m(m+2)$

\section{Conjecture 1:}

Consider all the number of the numbers of the form $(6 n-1)$ and $(6 n+1)$ noted A. The number of elements of $\mathrm{A}$ is a.

Consider all the prime numbers and the squares of the prime numbers noted set $\mathrm{B}$. The number of elements of $\mathrm{B}$ is $\mathrm{b}$.

We conjecture that $\mathrm{b} / \mathrm{a}$ is the twin prime constant.

Attempt to demonstrate: some curiosities

Let $\mathrm{n}$ be a natural nonzero. Consider the one hand the set of numbers of the form $(6 n-1)$ on the one hand and of the form $(6 n+1)$ on the other. Numbers of the form $(6 n-1)$ are in Table 1 . Numbers of the form $(6 n+1)$ are in Table 2 . 
Table I: The integers of the form $(6 n-1)$ up to 299

\begin{tabular}{|l|l|l|l|l|}
\hline 5 & 11 & 17 & 23 & 29 \\
\hline 35 & 41 & 47 & 53 & 59 \\
\hline 65 & 71 & 77 & 83 & 89 \\
\hline 95 & 101 & 107 & 113 & 119 \\
\hline 125 & 131 & 137 & 143 & 149 \\
\hline 155 & 161 & 167 & 173 & 179 \\
\hline 185 & 191 & 197 & 203 & 209 \\
\hline 215 & 221 & 227 & 233 & 239 \\
\hline 245 & 251 & 257 & 263 & 269 \\
\hline 275 & 281 & 287 & 293 & 299 \\
\hline
\end{tabular}

In red the twin primes

In blue primes not elements of pairs of twin primes

Black (not bold, not underlined) numbers not primes

Table II: The integers of the form $(6 n+1)$ up to 301

\begin{tabular}{|l|l|l|l|l|}
\hline 7 & 13 & 19 & $\underline{\mathbf{2 5}}$ & 31 \\
\hline 37 & 43 & $\underline{\mathbf{4 9}}$ & $\mathbf{5 5}$ & 61 \\
\hline 67 & 73 & 79 & 85 & 91 \\
\hline 97 & 103 & 109 & 115 & $\underline{\mathbf{1 2 1}}$ \\
\hline 127 & 133 & 139 & 145 & 151 \\
\hline 157 & 163 & $\underline{\mathbf{1 6 9}}$ & 175 & 181 \\
\hline 187 & 193 & 199 & 205 & 211 \\
\hline 217 & 223 & 229 & 235 & 241 \\
\hline 247 & 253 & 259 & 265 & 271 \\
\hline 277 & 283 & $\underline{\mathbf{2 8 9}}$ & 295 & 301 \\
\hline
\end{tabular}

In red the twin primes

In blue primes not elements of pairs of twin primes

Black (not bold, not underlined) numbers not primes

But in black and highlighted in bold writing: squares of primes

\section{Comments:}

The density of twin prime numbers in Table I is 0.36 . It is the same for Table II.

The density of primes not elements of twin prime pairs of Table I is 0.30 . That in Table II is 0.20 . Thus the density of prime numbers in Table I is 0.66 . That in Table II was 0.56 .

Note a peculiarity of Table II. Indeed it contains squares of prime numbers. So they are almost primes specific numbers. I call them almost-primes particulary numbers. Their density is 0.10. Which is exactly the gap of Table II with Table I in regard to prime numbers. If, by extrapolation we put them in the group of prime numbers we find the density of 0.66 .

The twin prime constant is estimated to $0.66016181584686957392781211001455577 \ldots$ It is some similar to the density of primes mentioned above (discussed above). 


\section{References:}

[1] Jean-Paul Delahaye. Merveilleux nombres premiers. Voyage au coeur de l'arithmétique , Éditions Belin/Pour la science, Paris, 2000, (Bibliothèque scientifique) 336 p. - 155 F.

[2] Jean-Paul Delahaye. Jeux mathématiques et mathématiques des jeux, Éditions Pour la science, Paris, 1998, (Bibliothèque Pour la science) 141 p. - 95 F. 